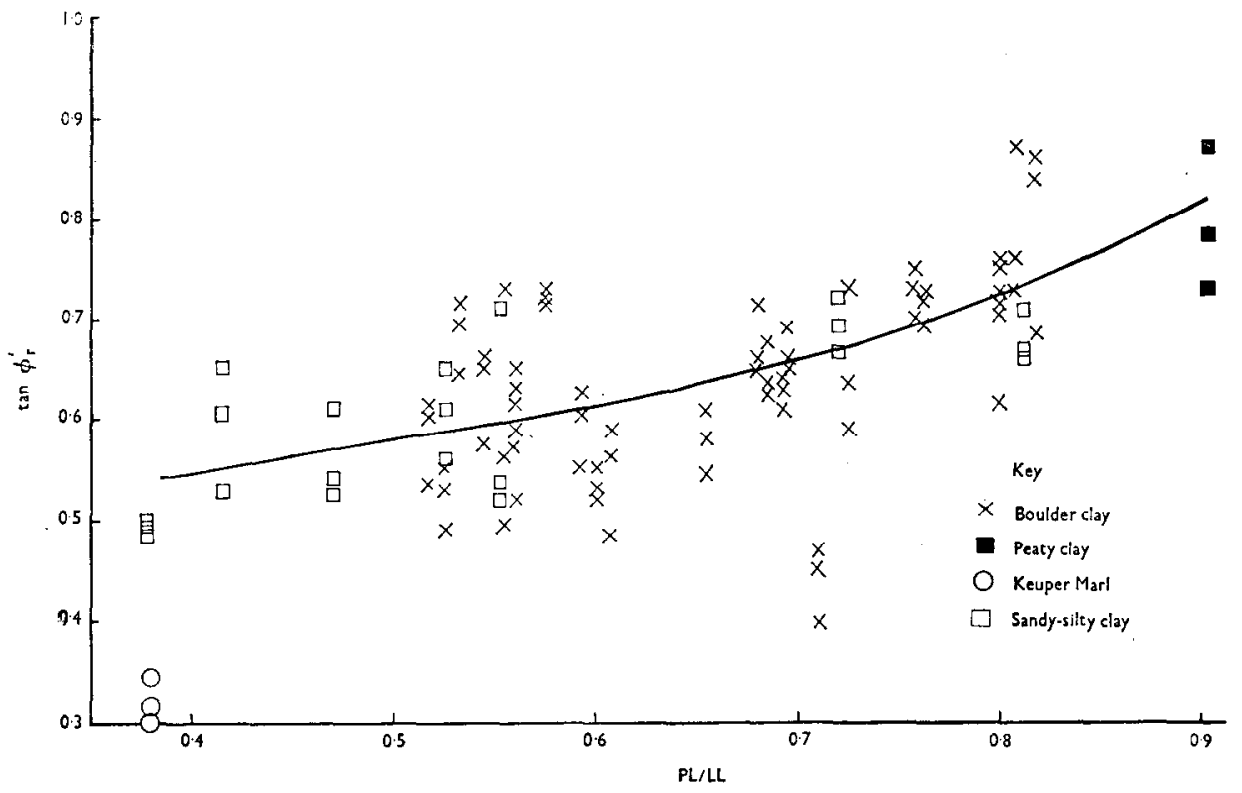

Fig. 1. Correlation between $\tan \phi_{r}^{\prime}$ and the ratio of plastic limit to liquid limit

residual cohesion intercept. Although the present plot was based on the assumption of $C_{\mathrm{r}}^{\prime}=0$ we believe that if the index properties for each residual shear box test were determined and the value of $C_{r}^{\prime}$ were taken into account the amount of scatter would have been greatly reduced.

Excluding the results of Keuper marl and one of the boulder clay samples, the figure shows a trend of increasing $\tan \phi_{\mathrm{r}}^{\prime}$ for increasing PL/LL. One possible reason for $\tan \phi_{\mathrm{r}}^{\prime}$ being very low for the Keuper marl sample may be that the silt-sized particles of Keuper Marl, which consist of conglomerated clay-sized particles, are broken up in the shear strength test (especially as in this case the test was carried out at fairly high normal pressures, $500-700 \mathrm{kN} / \mathrm{m}^{2}$ ), whilst in the liquid and plastic limit tests they remain intact. This would result in the LL and PL tests indicating that the Keuper marl particles are silt-sized whilst the shear strength test would be affected to some extent by the properties of the clay-sized particles.

\title{
Structural behaviour of residual soils of the continually wet Highlands of Papua New Guinea
}

\author{
WALLACE, K. B. (1973). Géotechnique 23, No. 2, 203-218.
}

\section{P. K. De and B. Furdas, Sir William Halcrow and Partners, Aberaman, Aberdare, Glamorgan}

Wallace is to be commended for his contribution on the structural behaviour of residual soils and the interpretation of the test results in terms of a structural model of the soil. However, every simplified structural model of soils has its limitations and the Author's model is by no means an exception.

We should like to take the opportunity of modifying the structural model to take into account certain physical properties of the residual soils and explain the behaviour of the soil in terms of 


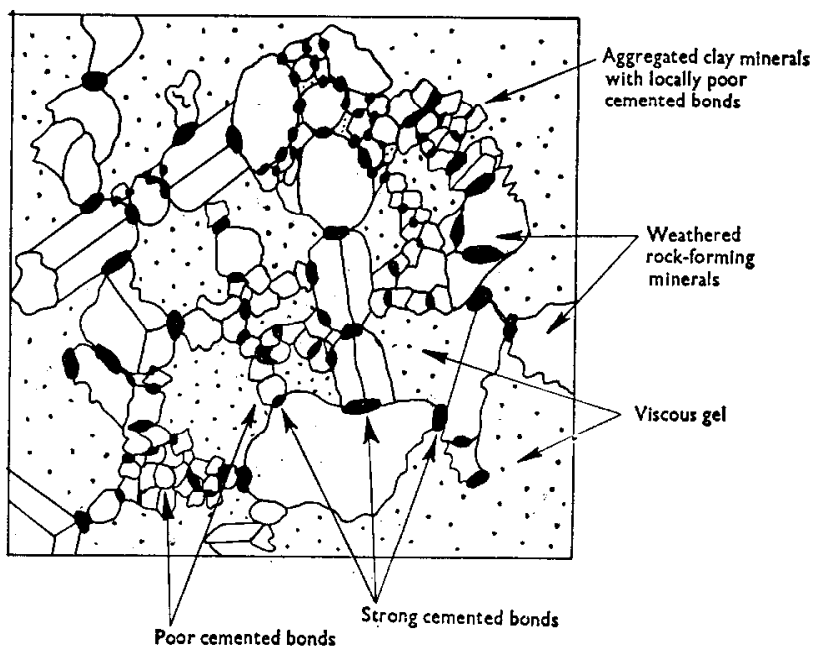

Fig. 1. Modified idealized residual soil structure

the reported shear strengths and compressibilities. Fig. 1, which shows the modified idealized residual soil structure is, however, not free from limitations and we are fully aware of this. It has been drawn to look very similar to that reported by the Author for the sake of comparison.

The results given in the Author's Table 5 of sieve analyses performed on natural and dried samples of soils 1 and 3 show that, on drying of the samples, large proportions of the silt and clay particles become aggregated to form sand-sized particles. The effect of drying the samples would therefore be to increase the strength of the cemented bonds and hence increase the percentage of aggregated sand-sized particles which were not dispersed by treatment with sodium hexametaphosphate. From this argument it can be concluded that many of the sandsized particles recorded for the natural samples could also be cemented aggregates of silt and clay particles. Indeed, some of the silt-sized particles could themselves be aggregates of clay particles, especially as our experience on dispersion of kaolinite particles indicates that the dispersion agent used, i.e. sodium hexametaphosphate, may not be powerful enough to break down the strong cemented bonds. External energy such as light ultrasonic vibration is needed in addition to the dispersing agent for better dispersion. One possible way of disinguishing true sand particles from aggregated sand-sized particles would be to perform a sedimentation analysis on particles of equal size. As the aggregated particles contain voids, they would have a lower specific gravity and would, therefore, sink more slowly.

We have reason to believe that the majority of the sand particles are in fact aggregates. From the Author's Table 1 it can be seen that the liquid limit increases as the sand percentage increases, which effectively means that by introducing more sand-sized particles into the system more voids are created. This can be visualized if the sand-sized particles are aggregates, as these would contain voids. However, if the sand-sized particles are true solid sand particles of the usual uneven spheroidal shape, it is difficult to visualize how such particles could introduce a very high degree of voids into the system.

In Fig. 2 of Wallace's paper an example of an $e-\log p$ graph for the soil is given, which shows that the compressibility is low for applied pressures below a certain critical pressure. For applied pressures greater than the critical pressure the compressibility is much higher. The relatively high percentage of clay fractions in the residual soils, as reported in Table 5, is a direct 
conservative indication of the amount of fine fractions and a product of the separation of the clay particles from its aggregated condition due to chemical treatment only. This suggests that the cemented bonds are not evenly strong and the existence of locally poor to verv poor bonds between the particles within the aggregated mass can not altogether be ruled out. Although the locally poor to very poor bonds can be disturbed and dislocated easily at low consolidation pressure, or at an early strain in the shearing process, the strongly cemented bonds remain virtually intact under such pressures. It is the distribution of the poor bonds that determine the shape of the $(e-\log p)$ graph. In a soil sample in which there is a large variation in strength of the cemented bonds, one would expect a gradual increase of compressibility with applied pressures, as the weaker cemented bonds would be broken down at much lower applied pressure. Although the nature of the strong cemented bonds cannot be estimated easily, it can be fairly assumed that the critical pressure of a consolidated test is an indirect measure of the relative strength of the strong bonds.

For the sake of simplicity, let us assume the existence of pesudo-domain structures within the soil. The strength of the cemented bonds within each domain would be generally stronger than between each individual domain. The stress-strain curve for the soaked undistributed sample at $\sigma_{n}^{\prime}=5 \mathrm{lbf} / \mathrm{in}^{2}$ normal pressure showed a high degree of brittleness. This can be explained in terms of the cemented bonds and pseudo-domain structures. At the very low applied normal pressure of $5 \mathrm{lbf} / \mathrm{in}^{2}$, i.e. about $0.3 \mathrm{tf} / \mathrm{ft}^{2}$, the sample presumably behaved elastically and as the shearing continued the inter-domain weak bonds became dislocated. The microscopic shear discontinuity would be irregular, less defined and thin. The interlocking of the pseudo-domains bordering the shear discontinuity would build up at an early strain, and as the confined pressure was low it would result in volume increase. As it approached the residual condition the shear discontinuity across the specimen would be continuous thus releasing the interlocking stress.

However, in the case of the sample tested at $\sigma_{n}^{\prime}=15 \mathrm{lbf} / \mathrm{in}^{2}$, i.e. about $1 \mathrm{tf} / \mathrm{ft}^{2}$, presumably the poor bonds became disturbed and dislocated at the relatively high consolidation pressure. During the shearing process, presumably the confining pressure was too great for the pseudodomains to ride over one another and so the strong bonds within the domains sheared.

Finally, we should like to add a comment on the effect of drying on permeability. Did the Author consider the possibility of piping action due to the aggregation of inter-domain structures? We believe that the viscous gel plays an important role in control of permeability. 